\title{
Fundamental Limits on Energy Transfer and Circuit Considerations for Piezoelectric Transformers
}

\author{
Anita M. Flynn and Seth R. Sanders, Member, IEEE
}

\begin{abstract}
This work investigates fundamental limits on electromechanical energy conversion capacity of piezoelectric transformers by considering a work cycle analysis. Fundamental limitations in a lossless piezoelectric transformer are imposed by maximum electric field strength, maximum surface charge density, maximum stress, and maximum strain. For the lossless case, our analysis indicates that the mechanical stress limit is the effective constraint in typical PZT materials. For a specific PZT-5H sample considered, a mechanical stress-limited work cycle indicates that this material can handle $330 \mathrm{~W} / \mathrm{cm}^{3}$ at $100 \mathrm{kHz}$.

A second direction this work has taken has been an investigation into a soft-switching drive and control circuit, that does not require any magnetic components. The theory of operation of softswitching resonant drive circuitry is discussed, and experimental results on a soft-switching inverter incorporating no magnetic components are reported.
\end{abstract}

Index Terms-Piezoelectric, soft-switching, transformer, work cycle.

\section{INTRODUCTION}

$\mathbf{P}$ OWER electronic circuits have conventionally been based on magnetic technology and until recently have not been part of the tide of miniaturization and integration advances from which signal-processing integrated circuits have benefited. In many power electronic systems today, the magnetic components remain as the bulkiest and costliest components. In an effort to achieve miniaturization, we are investigating alternative technologies to magnetics for provision of reactance, galvanic isolation and voltage/current scaling (transformer function). Specifically, we are investigating acoustic coupling in place of inductive coupling to create electro-mechanical transformers.

Acoustic coupling can be achieved through piezoelectric or magnetostrictive means. A piezoelectric transformer offers the potential advantages of a thinner profile than a magnetic transformer, and a simpler manufacturing process due to the lack of windings. Application areas range from battery-powered consumer electronics and cellular telephones to wearable computers and head-mounted displays. The driving application at present for a piezoelectric transformer is the step-up transformation required to power a cold cathode fluorescent lamp in a flat panel display from a laptop computer battery. These cold cathode fluorescent lamps require $1000 \mathrm{~V}$ to start and

Manuscript received January 17, 2000; revised May 2, 2001. Recommended by Associate Editor K. Ngo.

A. M. Flynn is with the MicroPropulsion Corporation, Berkeley, CA 94704 USA (e-mail: aflynn@micropropulsion.com).

S. R. Sanders is with the Electrical Engineering and Computer Science Department, University of California, Berkeley, CA 94720 USA (e-mail: sanders@eecs.berkeley.edu).

Publisher Item Identifier S 0885-8993(02)02167-1.
400-500 $\mathrm{V}$ to run at a $1-5 \mathrm{~W}$ power level and have an input impedance on the order of hundreds of kilohms, a good match for the piezoelectric transformer.

While piezoelectric transformers were originally developed in the United States in the 1950s [1], [2], they were not commercially pursued to a large extent due to poor materials reliability and competition from magnetic flyback transformers for cathode ray tubes. Recently, a number of researchers, mainly in Japan, have begun new efforts at producing compact, efficient, bulk-ceramic piezoelectric transformers for portable applications, most notably for laptop flat panel displays. These efforts have yielded reported efficiencies from $82 \%$ [3] to as high as $92 \%$ [4].

In this paper, we discuss fundamental limits of energy density and power throughput of an ideally lossless piezoelectric element based on materials considerations and a work cycle analysis. The work cycle considered is shown to be consistent with a square-wave voltage drive. However, such a hard-switched drive scheme in an inverter circuit leads to considerable switching losses as the static input capacitance of a piezoelectric transformer can be large. We subsequently describe a strategy for achieving soft-switching, and report on an inverter circuit that we have built which demonstrates soft-switching and high efficiency without the use of magnetic components.

\section{ElECTROMECHANICAL POWER TRANSFORMATION- FUNDAMENTAL LIMITS}

A piezoelectric transformer works by using the direct and converse piezoelectric effects to acoustically transform power from one voltage and current level to another. That is, power is converted electromechanically through a vibrating piezoelectric structure rather than electromagnetically as in an inductive transformer. Electromechanical coupling, in one dimension, is described by the piezoelectric constitutive relations [6]

$$
\begin{aligned}
& T_{3}=\left(D_{3}-\varepsilon_{33}^{T} E_{3}\right) / d_{33} \\
& S_{3}=s_{33}^{E} T_{3}+d_{33} E_{3} .
\end{aligned}
$$

The convention here is that the poling direction is taken as the 3-direction in a 1-2-3 labeled orthogonal coordinate system. Variable $E_{3}$ is the electric field strength applied in the direction the material was poled, while $D_{3}$ is the resulting surface charge density. Variables $T_{3}$ and $S_{3}$ are the reduced matrix notation representations for the stress tensor component, $T_{33}$, and strain tensor component, $S_{33}$, respectively. Parameter $s_{33}^{E}$ is the compliance taken at constant electric field, while $\varepsilon_{33}^{T}$ is the permittivity taken at constant stress, and $d_{33}$ is the piezoelectric coupling coefficient. 


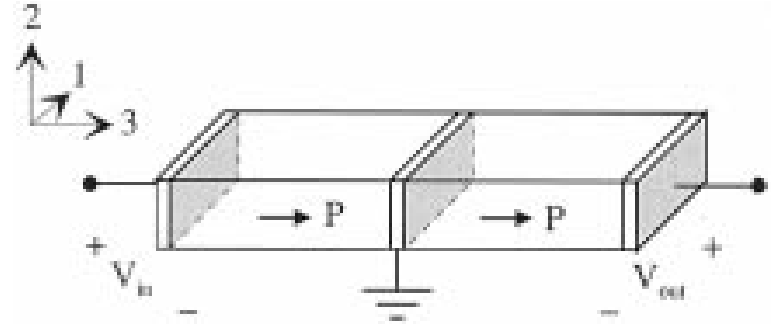

(a)

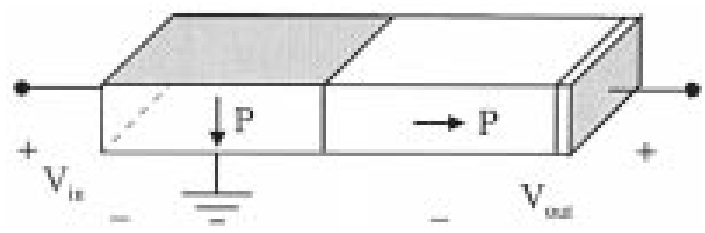

(b)

Fig. 1. Both transformers shown here are long thin bars of piezoelectric material driven in a longitudinal mode resonance (displacements in the 3-direction) [1]. (a) Two segments are poled as shown and electroded faces perpendicular to the poling directions provide contacts for the input and output voltages. (b) The input segment is poled transversely to the longitudinal mode displacements, utilizing the $d_{31}$ effect $\left(d_{31}=d_{32}\right)$. The generator segment uses $d_{33}$ coupling, a larger effect than $d_{31}$, which assists in creating larger voltage amplification.

Rosen first described piezoelectric transformers in [1], [2], and two of the simplest structures are shown in Fig. 1. In the transformers shown in the figure, the converse piezoelectric effect, in which an applied electric field produces a resulting strain in a body, is used to first convert an oscillating electric field applied to the left half of each bar into an extensional vibrational mode of the entire bar. If driven at resonance, standing-wave distributions of large amplitudes of stress and strain result. The resonantly amplified strain in the right half of each bar is then converted to a voltage across the output terminals by the direct piezoelectric effect. Depending upon the geometry and materials parameters, voltage amplifications of various magnitudes can be obtained, with associated step-downs in current levels.

\section{A. Power Density Calculation}

In designing piezoelectric transformers, it is useful to first investigate the fundamental limits of power density, i.e., the amount of power able to be transferred through the device per unit volume. During each cycle of electrical input, some amount of work is transformed in the left half of the bar from electrical energy to mechanical energy, and then similarly, in the right half of the bar, that same mechanical energy is transformed back to electrical energy. The maximum power density could be limited by a number of mechanisms such as electric breakdown strength, maximum surface charge density (i.e., saturation), maximum stress, maximum strain, or depoling due to electric fields. To address the power density issue, we consider an infinitesimal cellular element situated at a point in one half of a piezoelectric transformer, such as a point in the left half of the device in Fig. 1(a).

We determine the power density by examining a work cycle. Let us start by assuming a lossless system that is in state 1 of its work cycle (point 1 of Fig. 2). The structure is then blocked mechanically as the electric field is reduced first to $E_{3}=0$ and

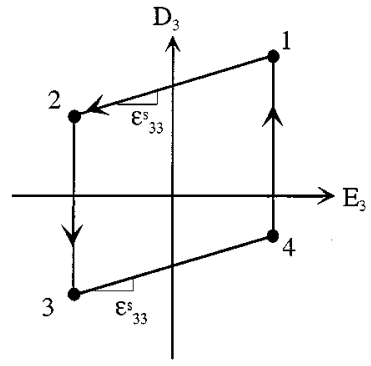

(a)

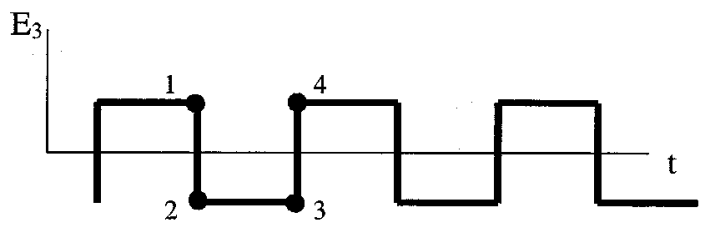

(c)

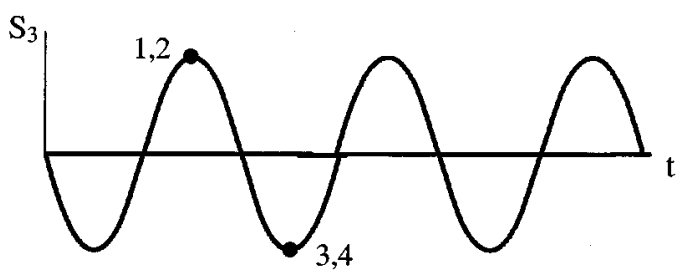

(d)
Fig. 2. Work cycle viewed in both the electrical and mechanical domains. As the work cycle is traversed, the area inside each parallelogram is the energy transformed from electrical to mechanical energy or vice versa. (a) Electrical domain. (b) Mechanical domain. (c) $E_{3}$ versus time. Points 1, 2, 3, and 4 represent the square-wave drive corresponding to the respective points in the work cycle of (a) and (b) when the piezoelectric structure is cycled at its resonant frequency. (d) $S_{3}$ versus time. The strain is at its maximum positive value during the instantaneous time that the electric field moves from its maximum positive value at position 1 to its most negative value at position 2 . Conversely, the strain is at its most negative value when $E_{3}$ transitions from its most negative value at state 3 to its maximum positive value at state 4 .

then to some negative value (point 2). At this point, the field is held constant and the mechanical block is removed, whereupon the structure moves from a state of positive strain to a state of negative strain (point 3), and the system has performed mechanical work in moving from point 2 to point 3 . The element is then mechanically blocked again in this new strain state and the electric field is increased (point 4). The electric field is then held constant and the mechanical block is removed, moving the system back to its original state of positive strain (point 1). The area enclosed by the work cycle represents the transformed energy density. Specifically, the area enclosed by the parallelogram in Fig. 2(a) represents the supplied electrical energy (density), while the area enclosed by the parallelogram in Fig. 2(b) represents the mechanical work (density). Note that these two areas are equal for this ideal lossless work cycle. The cycle is repeated periodically and the power throughput density is equal to the energy density multiplied by the frequency of operation.

We note that the trajectory of the work cycle of Fig. 2(a) and (b) is actually consistent with a square-wave voltage drive, as readily achieved with a square-wave inverter, or some variant thereof. Fig. 2(c) illustrates such a square wave drive. In this case, operation could be near resonance, where the voltage drive is switched from maximum (point 1 of Fig. 2) to minimum 


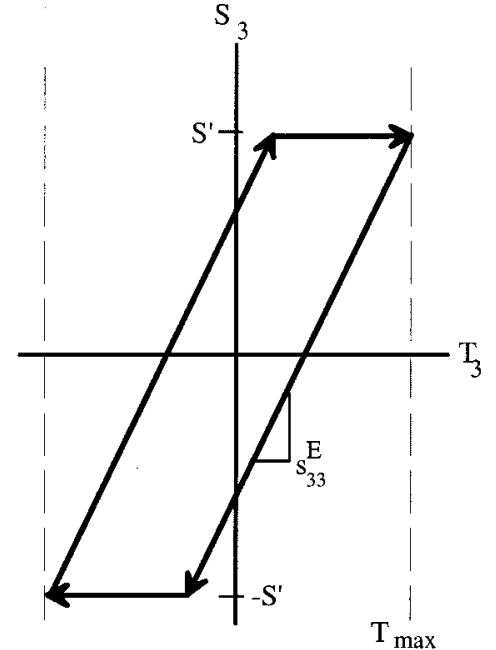

Fig. 3. Maximum energy density for a stress-limited material can be calculated by maximizing over $S^{\prime}$ the area of the parallelogram enclosed by the work cycle.

(point 2), when the strain, and corresponding displacement, is at its positive extreme. Conceptually, the voltage transition occurs instantaneously, and no change in the strain state occurs during this transition. Then the voltage drive is held constant at its minimum while the inertia of the resonant motion carries the transition from point 2 to point 3 , thus changing from a positive strain state to a negative strain state, as is illustrated in Fig. 2(d). The respective transitions from point 3 to point 4 and from point 4 to point 1 of Fig. 2 occur analogously to the transitions from point 1 to point 2 and from point 2 to point 3 , consistent with the symmetry of the trajectory.

Maximum power throughput depends on the maximum values of electric field, charge density, stress and strain which can be achieved with a given material. We consider the lossless case here and thus the computed power densities do not reflect thermally limited power transmission. In practice, steady state power density may also be limited by temperature rise due to internal losses [10]. For the lossless case, the maximum-area work cycle is bounded by one or more of the material's limits, $D_{\max }, E_{\max }, S_{\max }$ or $T_{\max }$. To explore the largest feasible work cycle, we pick a corner corresponding to maximum values in either domain, such as $\left(E_{\max }, D_{\max }\right)$ and map this state into the $S-T$ domain with the one-dimensional piezoelectric constitutive relations (1), (2).

For an example material such as the PZT-5H ceramic [5], specified in Table I, the point $\left(E_{\max }, D_{\max }\right)$ maps outside the $T_{\max }, S_{\max }$ bounding box. The bounding box is the region in the stress-strain plane that will not cause fracture. The material is thus mechanical-field-strength limited, i.e., it is either maximum stress limited or maximum strain limited. Assume the material is stress limited. From known values of $T_{\max }$ and slope $s_{33}^{E}$, a value of strain, $S^{\prime}$, can be found which maximizes the enclosed area, where the enclosed area is the energy transformed per unit volume. Fig. 3 shows the construction of the maximum-area work cycle for a stress-limited material. For a maximum stress of $T_{\max }$, and compliance at constant electric field, $s_{33}^{E}$, the area of the parallelogram (energy density) is

$$
W=2 S^{\prime}\left(2 T_{\max }-2 S^{\prime} / s_{33}^{E}\right) \quad\left(\text { Joules } / \mathrm{m}^{3}\right) .
$$
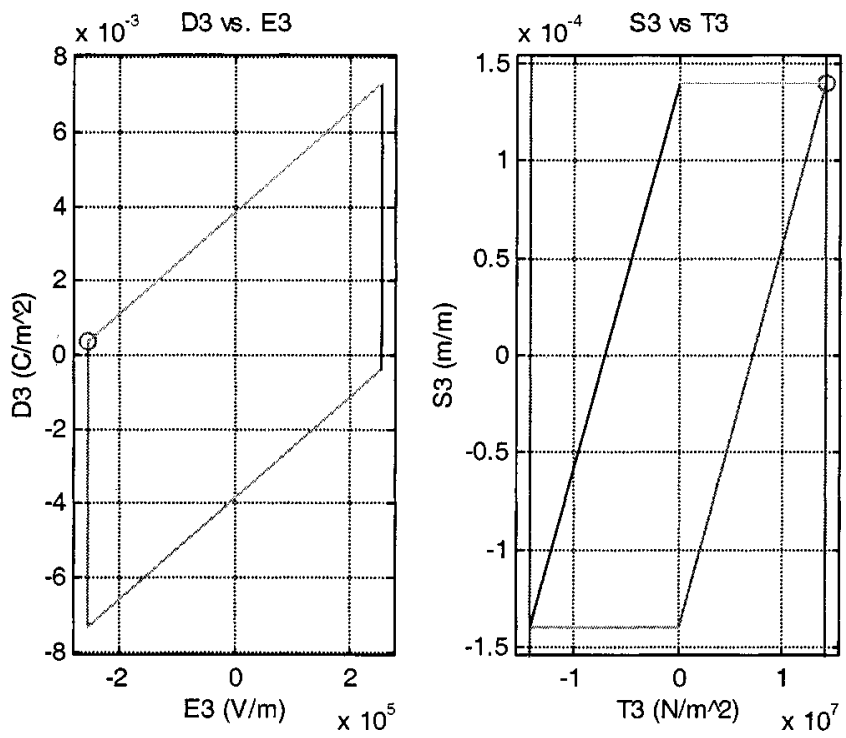

Fig. 4. Work cycle for stress-limited PZT-5H yields an energy density of $3920 \mathrm{~J} / \mathrm{m}^{3}$

Maximizing the area with respect to $S^{\prime}$ yields the optimum strain, $S^{*}$

$$
S^{*}=1 / 2\left(s_{33}^{E} T_{\max }\right) .
$$

This value of $S^{*}$ must be checked to ensure that it is less than $S_{\max }$. If so, this strain yields a maximum energy density of

$$
W_{\max }=s_{33}^{E} T_{\max }^{2} \quad\left(\text { Joules } / \mathrm{m}^{3}\right) .
$$

Maximum power density corresponds to maximum energy density per unit time, yielding

$$
P_{\max }=f W_{\max }=f s_{33}^{E} T_{\max }^{2} \quad\left(\text { Watts } / \mathrm{m}^{3}\right)
$$

where $f$ is the frequency at which the work cycle is traversed.

Fig. 4 illustrates the maximum area stress-limited work cycle for the example PZT-5H material with the characteristics shown in Table I. Note that maximum values for electric field, charge density, and strain are not exceeded. The calculated energy density from (5) is $3920 \mathrm{~J} / \mathrm{m}^{3}$. The resulting maximum power density from (6), in the lossless case, at $100 \mathrm{kHz}$ is thus $330 \mathrm{~W} / \mathrm{cm}^{3}$. For a ceramic piece $0.5 \mathrm{~mm}$ thick, this translates to $16.5 \mathrm{~W} / \mathrm{cm}^{2}$. As previously mentioned, this calculation is not based on a thermal limit, and hence may not provide a steady state maximum power density. Work addressing thermal limitations of piezoelectric transformers can be found in [10].

\section{Magnetics-Free Soft-Switched Drive}

The preceding analysis was based on a square-wave drive, applied exactly at resonance. As reported in the literature [7]-[9], the impedance at the input terminals of the piezoelectric transformer is actually inductive at a frequency just above resonance, as is the case with a conventional series resonant $L-C$ tank circuit. Fig. 5(a) shows a simplified equivalent circuit model of a piezoelectric transformer, corresponding to a model that accounts only for the principle resonant mode. Here, 
TABLE I

PZT-5H MATERIAL CONSTANTS

\begin{tabular}{llll}
\hline $\mathrm{K}^{\mathrm{T}}$ & Relative dielectric constant & 3250 & \\
$\varepsilon_{33}{ }^{\mathrm{T}}=\varepsilon_{0} \mathrm{~K}^{\mathrm{T}}{ }_{3}$ & Dielectric constant at constant stress & $2.88 \times 10^{-8}$ & $\mathrm{~F} / \mathrm{m}$ \\
$\mathrm{d}_{33} \mathrm{E}$ & Piezoelectric strain coefficient & $550 \times 10^{-12}$ & $\mathrm{~m} / \mathrm{V}$ \\
$\mathrm{c}_{33} \mathrm{E}$ & Elastic modulus at constant E field & $5.0 \times 10^{10}$ & $\mathrm{~N} / \mathrm{m}^{2}$ \\
$\mathrm{~S}_{33} \mathrm{E}$ & Elastic compliance at constant E field & $2.0 \times 10^{-11}$ & $\mathrm{~m} / \mathrm{N}$ \\
$\rho$ & Density & 7700 & $\mathrm{~kg} / \mathrm{m}^{3}$ \\
$\mathrm{E}_{\max }$ & Initial depolarization field & $3.0 \times 10^{5}$ & $\mathrm{~V} / \mathrm{m}$ \\
$\mathrm{S}_{\max }$ & Maximum strain & 0.002 & \\
$\mathrm{D}_{\max }$ & Maximum charge density & 0.4 & $\mathrm{C} / \mathrm{m}^{2}$ \\
$\mathrm{~T}_{\max }$ & Maximum stress & $1.4 \times 10^{7}$ & $\mathrm{~N} / \mathrm{m}^{2}$ \\
\hline
\end{tabular}

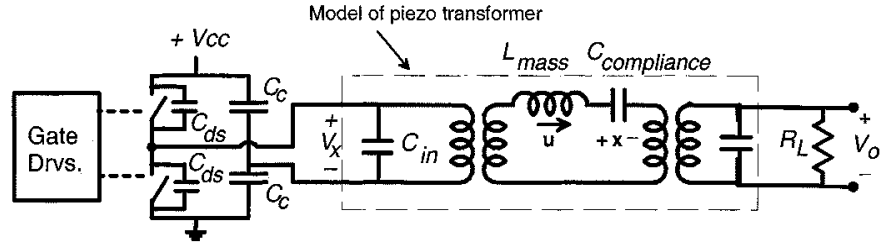

(a)

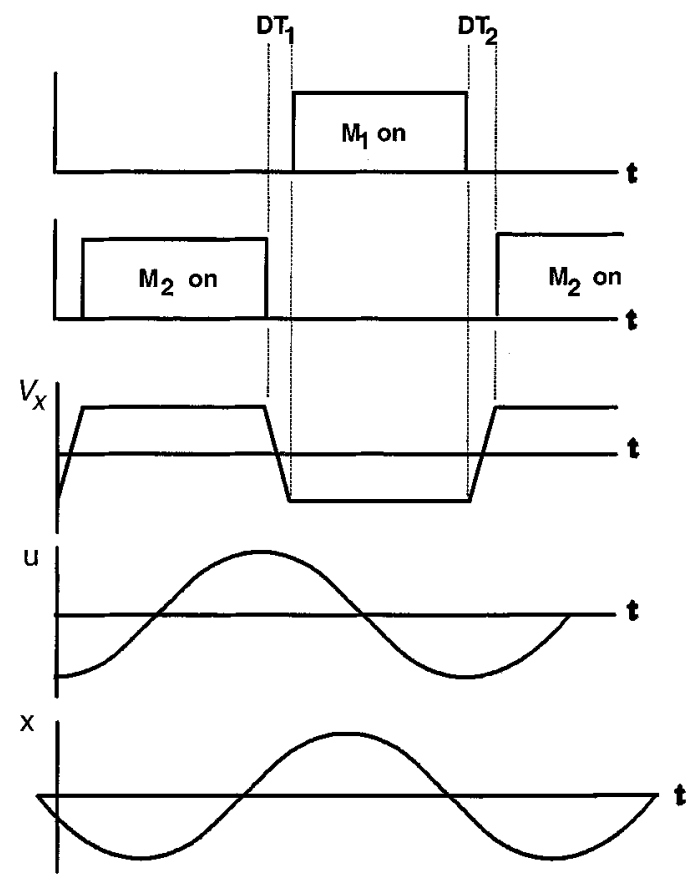

(b)

Fig. 5. (a) Inverter circuit for driving the primary of a piezoelectric transformer. The equivalent circuit model for the piezoelectric transformer is enclosed in the dashed lines. (b) Drive waveforms consistent with zero-voltage switching.

the principle resonant mode is the extensional mode of the piezoelectric device that coincides with the drive frequency. Transformers of this type have been used in the fundamental and second extensional resonant modes [12]. In the model of Fig. 5(a), the input capacitance corresponds to static electrical capacitance with the transformer mechanically blocked. The compliance corresponds to a lumped parameter value for the principle resonant mode, and the mass corresponds to a lumped parameter value for the same resonant mode. Ideal transformers in Fig. 5(a) indicate coupling between electrical and mechanical domains.

The resonant characteristic of the piezoelectric transformer can be used to overcome switching losses associated with switching or commutating the static input capacitance if the transformer is driven above resonance. That is, by operating the inverter at a frequency slightly above resonance, and by providing for an appropriate deadtime on each inverter switching transition, it is possible to obtain resonant soft-switching. This constitutes a variation of the work cycle introduced in the preceding section and is illustrated in Fig. 5(b), and discussed below.

If a deadtime $\mathrm{DT}_{2}$ is provided after turning off $M_{1}$ and before turning on $M_{2}$, the transformer input capacitance, in combination with the mosfet drain-source capacitances, is free to be charged up through the resonant mechanical motion of the transformer device. Specifically, during this deadtime, the variable $\mathbf{u}$ in Fig. 5(a), representing velocity of the lumped parameter mass, is negative. The continuing mechanical motion causes a corresponding change in strain state and displacement $\mathrm{x}$, and a subsequent change in the charge-voltage state at the electrical terminals. The change in strain and displacement can easily be large enough to supply all the charge needed to drive the complete voltage transition from negative state to positive state during the deadtime, even considering the charge required by the transformer input capacitance and also the drain-source capacitances of the power mosfets. This resonant transition is analogous to that which is used to achieve zero-voltage switching in a half-bridge series resonant converter [13]. A complementary zero-voltage switching transition is effected during the deadtime $\mathrm{DT}_{1}$.

There is a subtle difference from the work cycle of Fig. 2 with the proposed soft-switching cycle. Specifically, in Fig. 2, the transition from points 1 to 2 occurs with the piezoelectric slab mechanically blocked, and this transition is effectively driven at the electrical port. In the proposed soft-switching cycle, the change in state of electric field occurs while the piezoelectric slab is in motion, and with the electrical terminals open-circuited. Thus, the soft-switching transition occurs with a constant value of charge density $\mathbf{D}$. An analogous complementary transition occurs from points 3 to 4 . 


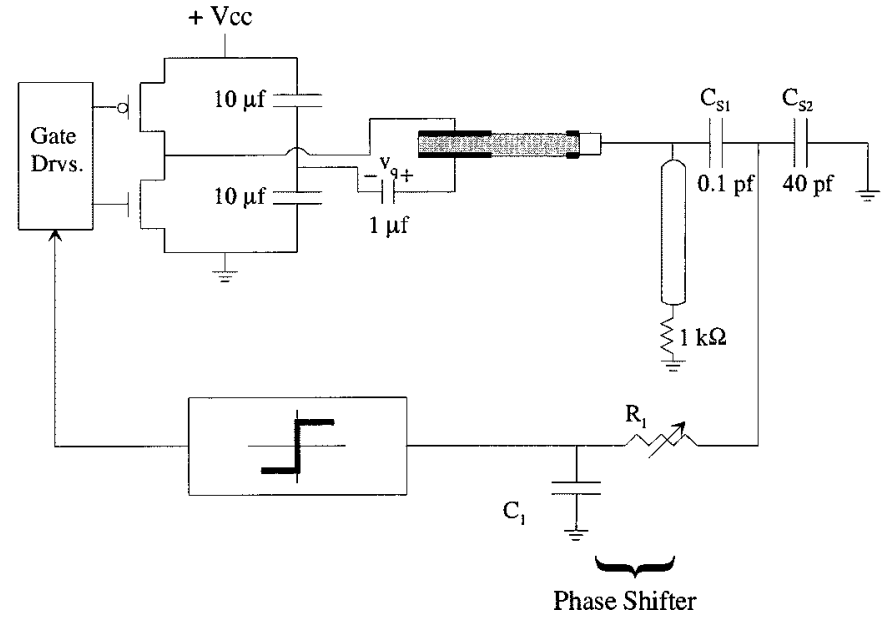

Fig. 6. Primary of the piezoelectric transformer is driven by a MOSFET halfbridge and the cold cathode fluorescent lamp is the load on the secondary. A feedback signal is tapped off the transformer secondary via a capacitive voltage divider and fed back to the gate drivers to create a self-oscillating system. The feedback signal is phase shifted such that a zero-crossing comparator produces the appropriately phased drive signal for the gate drivers.

\section{EXPERIMENTAL RESULTS}

An experimental setup was built to verify the proposed softswitching inverter scheme. The purpose was not to verify operation at maximum power density. Fig. 6 is a block diagram of a circuit realization of this inverter scheme that is self-oscillating. The power train is a half-bridge, always driven at 50\% duty cycle. The gate drive block provides gate drive for the power train, along with appropriately adjusted dead times. The remainder of the circuit is a very simple feedback scheme to cause the inverter to frequency lock just above a resonant frequency. This is explained in the sequel.

Capacitors $C_{S 1}$ and $C_{S 2}$ form a capacitive voltage divider to allow sampling the large output voltage without overloading this very high impedance terminal. The phase shifter network, $R_{1}-C_{1}$ adjusts the phase of the output signal, which is then fed to a comparator to provide the inverter drive signal. The use of the equivalent circuit model for the piezoelectric transformer, as previously introduced and shown in Fig. 7, is most convenient for understanding the operation. In this model, the capacitor, $C_{\text {compliance, }}$ represents the compliance, with state corresponding to strain. The inductor, $L_{\text {mass }}$, represents the mass, with state corresponding to mechanical velocity. From inspection of the electrical circuit representation and corresponding waveforms, it is clear that a steady state oscillation above resonance is consistent with the description. Roughly, $V_{o}$ lags the fundamental of $V_{x}$ by more than $90^{\circ}$ due to operation above resonance. The signal $V_{f}$ lags $V_{o}$ by an amount provided by the phase shifter, such that $V_{f}$ lags $V_{x}$ by 180 degrees. The power train (gate drivers and inverter) provides the final $180^{\circ}$ inversion function to effect a net $360^{\circ}$ of delay around the loop.

An heuristic argument for stability can be motivated by the Nyquist criteria and the describing-function analysis method [11]. For a steady-state oscillation, a loop gain of unity and phase shift of some integral multiple of $2 \pi$ is required. The phase condition is consistent with the indicated waveforms. The loop gain condition is achieved courtesy of the limiting behavior of the comparator nonlinearity. As the comparator input ampli-

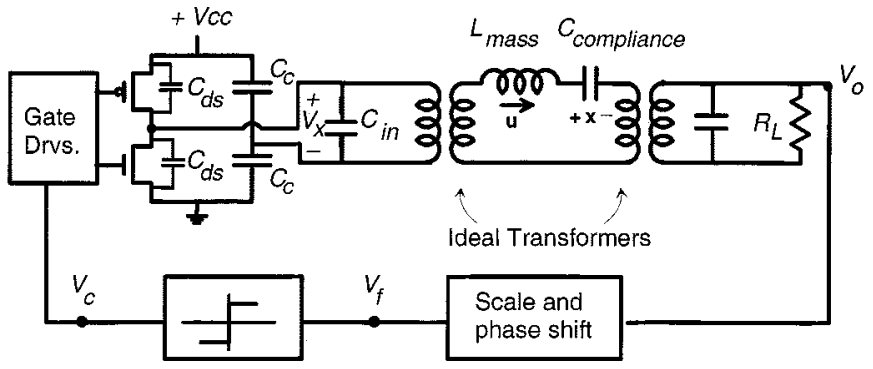

(a)

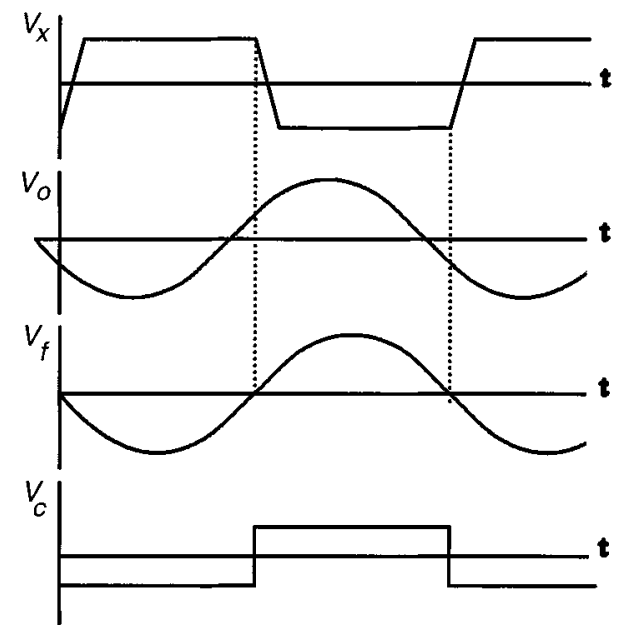

(b)

Fig. 7. Equivalent model of the piezoelectric transformer consists of two ideal transformers which model the mechanical-electrical and electrical-mechanical energy conversion processes respectively. $L_{\text {mass }}$ and $C_{\text {compliance }}$ represent the resonant characteristic of the transformer. During the deadtimes when both MOSFET's are off, the transformer impedance appears inductive, creating a current source that pulls or pushes charge off the static input capacitance of the transformer and also the parasitic capacitances at the MOSFET inverter node. Since the MOSFET's are relieved from supplying the power to do this, switching losses are averted and efficiency is increased relative to a hardswitched configuration.

tude scales up, the effective gain of the loop is reduced. Thus, from a Nyquist stability point of view, if the amplitude is perturbed upward, the gain is reduced, making the system "more stable" and thus resulting in an amplitude reduction. An analogous argument applies if the amplitude is perturbed downward.

A prototype of the circuit of Fig. 6 was constructed and used with a Rosen transformer of the type shown in Fig. 1(b). The objective was only to verify operation of the soft-switching drive circuit, and not to exercise the piezo transformer to its maximum power density. The piezoelectric material was PZT-4, and overall dimensions were $48 \mathrm{~mm} \times 8 \mathrm{~mm} \times 2 \mathrm{~mm}$. The second longitudinal extension mode resonance at $70 \mathrm{kHz}$ yielded best results. This was also noted in [12]. A small cold cathode fluorescent lamp was used as the load. Due to the relatively thick PZT sample, a relatively high voltage of about $45 \mathrm{~V}$ for the inverter supply was used to obtain a power output of about $500 \mathrm{~mW}$. Thinner transformers can operate efficiently with lower input voltages.

For the circuit implementation of Fig. 6, experimental primary voltage and charge waveforms are shown in Fig. 8(a). The waveform at top is $V_{x}$, the primary voltage applied by the inverter, at $20 \mathrm{~V} / \mathrm{div}$ and $2 \mu \mathrm{s} / \mathrm{div}$. Note the trapezoidal shape, indicative of the soft-switching action. The lower waveform in Fig. 8(a) is $V_{q}$, which is representative of the charge on the pri- 


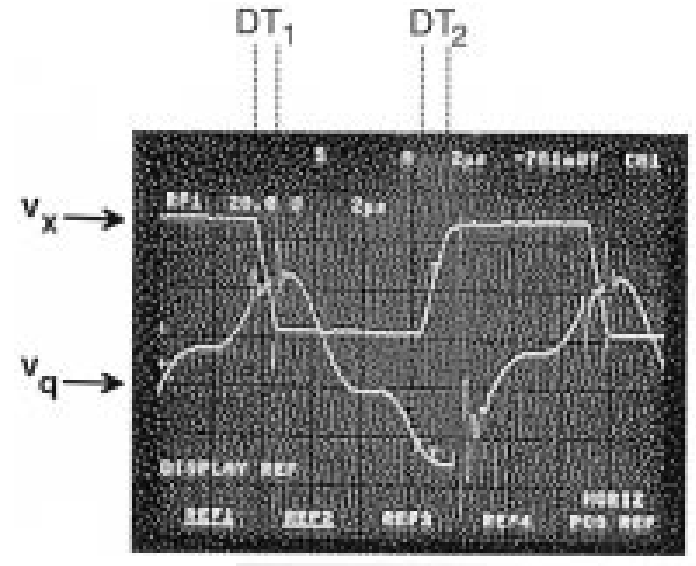

(a)

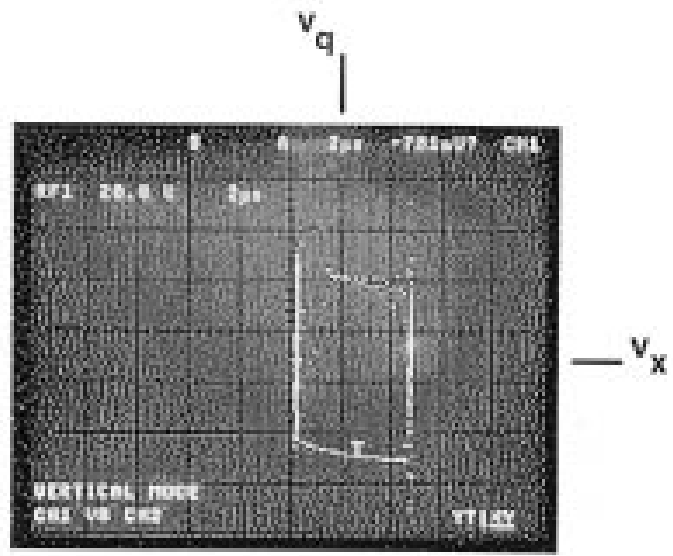

(b)

Fig. 8. (a) The trapezoidal waveform at the top is the voltage applied to the transformer primary, labeled $v_{x}$ in Fig. 7 . Note that during the deadtime, DT 2 , the voltage ramps up due to the inertia of the transformer causing a change in the charge-voltage state even when the primary is undriven. The vertical scale is $20 \mathrm{~V} /$ div and the horizontal scale is $2 \mu \mathrm{s} /$ div. The lower trace represents the charge at that node, measured as the voltage (labeled $v_{q}$ in Fig. 6) across a $1 \mu \mathrm{F}$ capacitor placed in series with the primary. The vertical scale of the lower trace is $50 \mathrm{mV} /$ div, or equivalently, $50 \mathrm{nC} / \mathrm{div}$. (b) Here, the two traces of (a) are plotted against each other with transformer primary charge, $v_{q}$, on the $y$-axis and primary voltage, $v_{x}$, on the $x$-axis, thus tracing out the electrical domain work cycle at the primary of the transformer. In comparing to Fig. 2(a), note that hard-switching was assumed there, whereas soft-switching is achieved here. The change in charge during a low-to-high voltage transition is approximately $1 / 3$ of a division decrease. That is, charge comes off the transformer primary to charge up the parasitic capacitance at the inverter node and swing the voltage up.

mary, obtained by sampling across a $1 \mu \mathrm{F}$ capacitor inserted in series with the primary. Scale for this waveform is $50 \mathrm{mV} / \mathrm{div}$, or equivalently, $50 \mathrm{nC} / \mathrm{div}$. Fig. 8(b) provides a charge-voltage phase portrait for these same waveforms, by displaying charge on the vertical $y$-axis and voltage on the horizontal $x$-axis. Note the close resemblance to the electrical work cycle of Fig. 2(a). The main difference is that in Fig. 8(b), as the voltage increases, the charge actually decreases. The charge decrease is exactly the charge required to swing the parasitic capacitance associated with the MOSFET inverter output. By inspection of Fig. 8(b), it is evident that this charge is about $15 \mathrm{nC}$, consistent with the charge needed to swing a parasitic output capacitance of about $330 \mathrm{pF}$ through $45 \mathrm{~V}$.

We note that if a hard-switched square-wave is applied at the primary, the switching loss is significant. A total capacitance of about $1 \mathrm{nF}$ needs to be switched-about $300 \mathrm{pF}$ associated with our inverter and approximately $700 \mathrm{pF}$ associated with the static input capacitance of the transformer. The resulting $C V^{2} f$ switching loss is then around $140 \mathrm{~mW}$ for a $45 \mathrm{~V}$ input voltage, and operation at $70 \mathrm{kHz}$. This comprises more than a $30 \%$ loss!

Fig. 9 shows the secondary electrical waveforms along with the primary voltage [as in Fig. 8(a)]. The sinusoid-like waveform is $v_{C_{s 2}}$, a scaled version of the output voltage obtained through a 400:1 capacitive divider. This is shown on a $1 \mathrm{~V} / \mathrm{div}$ scale, indicating an output voltage of about $1200 \mathrm{~V}$ peak. Note that the phase of this output voltage is related to the primary drive voltage, as shown in Fig. 5, and previously discussed. The third waveform represents the lamp current, sampled with a $1 \mathrm{k} \Omega$ resistor. This waveform is shown on a $1 \mathrm{~V} / \mathrm{div}$, or equivalently $1 \mathrm{~mA}$ /div, scale. Lamp current is thus about $2.4 \mathrm{~mA}$ peak. The phase shift between the lamp current and the transformer secondary output voltage is due to a small compensation capacitor of about $4 \mathrm{pF}$ placed in series with the lamp.

We note that accurate estimation of the output power from these waveforms is difficult. Consequently, we measured efficiency for the system (transformer and converter circuitry) by

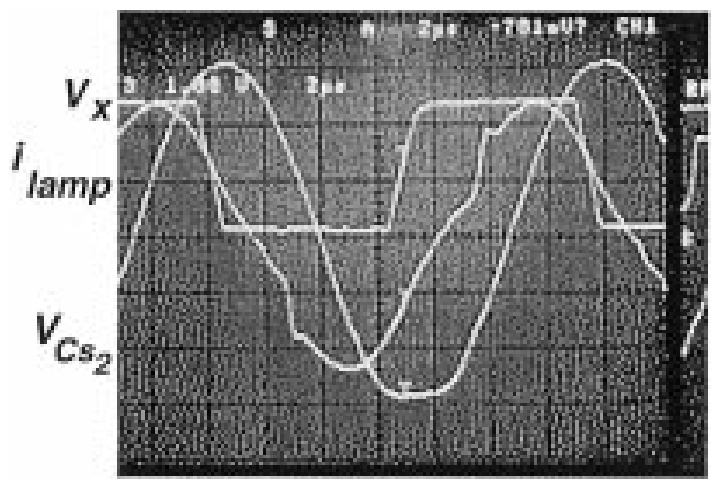

Fig. 9. The trapezoidal waveform, $v_{x}$, is shown again, as in Fig. 8, for reference. The smooth sine waveform is $1 / 400$ of the voltage at the transformer secondary. That is, this waveform is the voltage fed into the feedback circuit from the capacitive voltage divider attached to the transformer secondary. The vertical scale of this feedback signal is $1 \mathrm{~V} /$ div. The horizontal axis is again 2 $\mu \mathrm{s} /$ div. The third waveform represents the current through the lamp, measured across a $1 \mathrm{k} \Omega$ resistor (shown in Fig. 6) placed in series with the lamp. The vertical scale of the third waveform is $1 \mathrm{~V} / \mathrm{div}$, or equivalently, $1 \mathrm{~mA} / \mathrm{div}$.

TABLE II

EFFICIENCY MEASUREMENTS

\begin{tabular}{ll}
\hline $\mathrm{V}_{\text {in }}$ & $43.4 \mathrm{~V}$ \\
$\mathrm{I}_{\text {in }}$ & $8.7 \mathrm{~mA}$ \\
$\mathrm{P}_{\text {in }}$ & $376 \mathrm{~mW}$ \\
$\mathrm{R}_{\text {load }}$ & $216.3 \mathrm{k} \Omega$ \\
$\mathrm{I}_{\text {out }}$ & $1.7 \mathrm{~mA}$ \\
$\mathrm{~V}_{\text {load }}$ & $368 \mathrm{~V}$ \\
$\mathrm{P}_{\text {out }}$ & $313 \mathrm{~mW}$ \\
$\eta$ & $83 \%$ \\
\hline
\end{tabular}

replacing the fluorescent lamp with a resistive load in series with the $1 \mathrm{k} \Omega$ current sense resistor. Table II shows the measured results of output power dissipated in this load and the measured dc input power. The efficiency was determined to be $83 \%$. 


\section{CONCLUSION}

Piezoelectric transformers offer potentially impressive power density. We calculated the field-limited maximum power density and found that it was stress-limited. The peak power density for a PZT-5H sample was calculated to be $330 \mathrm{~W} / \mathrm{cm}^{3}$ at $100 \mathrm{kHz}$

We also showed that it is possible to effect soft-switching of a piezoelectric transformer in an inverter circuit without any magnetic components and to achieve efficiencies on the order of $83 \%$. Without soft-switching, it would be impossible to attain efficiencies above $70 \%$. Significantly, we experimentally verified that the entire driver for a cold cathode fluorescent lamp can be implemented without the need for any magnetic components. The manufacturing process for piezoelectric transformers can be much simpler than for electromagnetic transformers as no windings or assembled cores are required. The thin, flat form factor of the piezoelectric transformer further enhances this packaging consideration.

\section{REFERENCES}

[1] H. W. Katz, Ed., Solid State Magnetic and Dielectric Devices. New York: Wiley, 1959.

[2] C. Rosen, "Electromechanical Transducer," U.S. Patent 2974 296, Mar. 7, 1961.

[3] T. Zaitsu, T. Shigehisa, M. Shoyama, and T. Ninomiya, "Piezoelectric transformer converter with PWM control," in Proc. 1996 IEEE Appl. Power Electron. Conf. (APEC'96), 1996, pp. 279-283.

[4] S. Kawashima, O. Ohnishi, H. Hakamata, S. Tagami, A. Fukuoka, T. Inoue, and S. Hirose, "Third order longitudinal mode piezoelectric ceramic transformer and its application to high-voltage power inverter," in Proc. IEEE Ultrason. Symp., Cannes, France, 1994, pp. 525-530.

[5] Piezo Systems Technical Literature [Online]. Available: http://www.piezo.com

[6] B. Jaffe, W. R. Cook, and H. Jaffe, Piezoelectric Ceramics. New York: Academic, 1971.

[7] T. Ninomiya, M. Shoyama, T. Zaitsu, and T. Inoue, "Zero-voltageswitching techniques and their application to high-frequency converter with piezoelectric transformer," in Proc. 20th Ann. IEEE Conf. Ind. Electron., Bologna, Italy, 1994, pp. 1665-1669.

[8] M. Shoyama, K. Horikoshi, T. Ninomiya, T. Zaitsu, and Y. Sasaki, "Steady-state characteristics of the push-pull piezoelectric inverter," in Proc. Conf. Rec.28th Ann. IEEE Power Electron. Spec. Conf. (PESC'97), St. Louis, MO, 1997, pp. 715-721.

[9] P. J. M. Smidt and J. L. Duarte, "Powering neon lamps through piezoelectric transformers," in Proc. Conf. Rec. 27th Ann. IEEE Power Electron. Spec. Conf. (PESC96), Baveno, Italy, 1996, pp. 310-315.
[10] Y. Fuda, K. Kumasaka, M. Katsumo, H. Sato, and Y. Ino, "Piezoelectric transformer for cold cathode fluorescent lamp inverter," Jpn. J. Appl. Phys., vol. 86, pp. 3050-3052, May 1997.

[11] A. Gelb and W. E. V. Velde, Multiple-Input Describing Functions and Non-Linear System Design. New York: McGraw-Hill, 1968.

[12] T. Zaitsu, T. Inoue, O. Ohnisi, and A. Iwamoto, " $2 \mathrm{MHz}$ power converter with piezoelectric ceramic transformer," in Proc. IEEE INTELEC Conf., 1992, pp. 430-437.

[13] R. L. Steigerwald, "A comparison of half-bridge resonant converter topologies," IEEE Trans. Power Electron., vol. 3, pp. 174-182, Apr. 1988

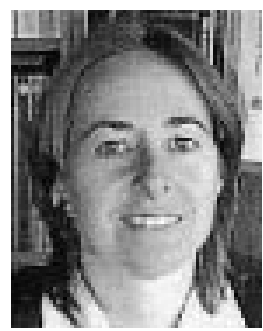

Anita M. Flynn received the B.S.E.E., M.S.E.E., and $\mathrm{Ph} . \mathrm{D}$. degrees from the Massachusetts Institute of Technology, Cambridge, in 1983, 1985, and 1995, respectively.

She spent two years at the University of California, Berkeley (UC Berkeley), as a Lecturer/Post-doctoral Assistant from 1995 to 1997, researching piezoelectric transformers. She is presently President of MicroPropulsion Corporation, specializing in consulting/contracting work in the areas of circuit design, power electronics, and MEMS. She was a Visiting Professor in the Mechanical Engineering Department, UC Berkeley, from 1999 to 2000.

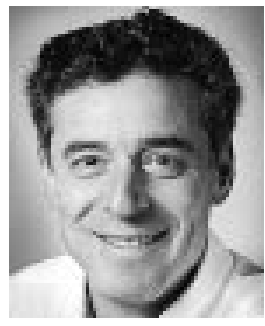

Seth R. Sanders (M'88) received the S.B. degree in electrical engineering and the S.B. degree in physics, the S.M. degree in electrical engineering, and the $\mathrm{Ph} . \mathrm{D}$. degree in electrical engineering, all from the Massachusetts Institute of Technology, Cambridge, in 1981,1985 , and 1989 respectively.

From 1981 to 1983, he was a Design Engineer at the Honeywell Test Instruments Division, Denver, CO. Since 1989, he has been on the faculty of the Department of Electrical Engineering and Computer Sciences, University of California, Berkeley, where he is presently Associate Professor. His research interests are in high frequency power conversion circuits and components, in variable speed ac motor drive systems, and in nonlinear circuit and system theory as related to the power electronics field. During the 1992 to 1993 academic year, he was on industrial leave with National Semiconductor in Santa Clara, CA.

Dr. Sanders received the NSF Young Investigator Award in 1993 and is a co-author on papers awarded prizes by the IEEE Power Electronics Society and the IEEE Industry Applications Society. He has served as Chair of the IEEE Technical Committee on Computers in Power Electronics, and as a Member-AtLarge of the IEEE PELS Adcom. 